\title{
Quantitative Measurements using Ultrasound Vector Flow Imaging
}

\author{
Jensen, Jørgen Arendt
}

Published in:

Proceedings of IEEE Ultrasonics Symposium

Link to article, DOI:

10.1109/ULTSYM.2016.7728672

Publication date:

2016

Document Version

Peer reviewed version

Link back to DTU Orbit

Citation (APA):

Jensen, J. A. (2016). Quantitative Measurements using Ultrasound Vector Flow Imaging. In Proceedings of IEEE Ultrasonics Symposium IEEE. https://doi.org/10.1109/ULTSYM.2016.7728672

\section{General rights}

Copyright and moral rights for the publications made accessible in the public portal are retained by the authors and/or other copyright owners and it is a condition of accessing publications that users recognise and abide by the legal requirements associated with these rights.

- Users may download and print one copy of any publication from the public portal for the purpose of private study or research.

- You may not further distribute the material or use it for any profit-making activity or commercial gain

- You may freely distribute the URL identifying the publication in the public portal

If you believe that this document breaches copyright please contact us providing details, and we will remove access to the work immediately and investigate your claim. 


\title{
Quantitative Measurements using Ultrasound Vector Flow Imaging
}

\author{
Jørgen Arendt Jensen \\ Center for Fast Ultrasound Imaging, Department of Electrical Engineering, \\ Technical University of Denmark, DK-2800 Lyngby, Denmark
}

\begin{abstract}
Duplex Vector Flow Imaging (VFI) imaging is introduced as a replacement for spectral Doppler, as it automatically can yield fully quantitative flow estimates without angle correction. Continuous VFI data over $9 \mathrm{~s}$ for 10 pulse cycles were acquired by a $3 \mathrm{MHz}$ convex probe connected to the SARUS scanner for pulsating flow mimicking the femoral artery from a CompuFlow 1000 pump (Shelley Medical). Data were used in four estimators based on directional transverse oscillation for velocity, flow angle, volume flow, and turbulence estimation and their respective precisions. An adaptive lag scheme gave the ability to estimate a large velocity range, or alternatively measure at two sites to find e.g. stenosis degree in a vessel. The mean angle at the vessel center was estimated to $90.9^{\circ} \pm 8.2^{\circ}$ indicating a laminar flow from a turbulence index being close to zero $(0.1 \pm 0.1)$. Volume flow was $1.29 \pm 0.26 \mathrm{~mL} / \mathrm{stroke}$ (true: $1.15 \mathrm{~mL} / \mathrm{stroke}$, bias: $12.2 \%$ ). Measurements down to $160 \mathrm{~mm}$ were obtained with a relative standard deviation and bias of less than $10 \%$ for the lateral component for stationary, parabolic flow. The method can, thus, find quantitative velocities, angles, and volume flows at sites currently inaccessible to spectral systems, and at much larger velocities and ranges than conventional systems without any angle correction making measurements less time-consuming and more correct.
\end{abstract}

\section{INTRODUCTION}

Spectral Doppler is currently used for quantitative measurements of peak velocities, resistive index, turbulence, and degree of stenosis. All have to be angle corrected and assumes a single angle. This is in general not valid for complex, pulsating flow through constrictions and tortuous vessels, where flow angles change over time and space. Measures based on a single angle can therefore be highly erroneous and lead to wrong diagnosis.

Current ultrasound systems use power Doppler or color flow mapping to identify places of flow, and subsequently uses spectral velocity estimation for determining quantitative measures [1], [2]. Here ultrasound pulses are emitted continuously in one direction and segments of data are Fourier transformed to yield the velocity distribution from which quantitative velocity measures can be found. The method has several drawbacks. The velocity is only found in the axial direction and must be angle corrected to yield velocity magnitude. Most often vessels are parallel to the skin surface and the beam-to-flow angle is close to $90^{\circ}$, making the angle correction unreliable and error prone. The angle correction also relies on a single angle over the full cardiac cycle, which is not correct for complex flow in many vessels [3], [4]. The spectral estimates also suffers from spectral broadening artifacts from the segmentation and windowing of the data [5]. A consistent over-estimation of peak and mean velocities are therefore often found. The maximum velocity detectable is limited by the pulse repetition frequency and the employed wavelength, which are fixed. This, in combination with the length of the segments used, gives the lowest velocity detectable and hence the velocity range, which can be estimated during a single measurement.

Transverse Oscillation (TO) vector flow imaging [6] can be used to remedy these problems. Continuous data for velocity estimation is available by pulsing in the same direction as for spectral systems similarly to the approach using plane waves in [7]. The continuous data enables average velocity estimates over many emissions, thus, reducing standard deviation (SD) of the estimates [8], [9]. The estimates yield both the correct velocity magnitude and angle, and they can be used for reliable estimation of peak velocities, volume flow, velocity profiles, and the estimation of turbulence as shown in Section IV. This paper uses directional TO (DTO) [10] as described in Section II to increase accuracy, and this can also be used to increase the peak detectable velocity by a factor of 2 to 8 compared to spectral systems. The velocity range can even be adapted retrospectively and dynamically over time to the actual velocity. Examples from flow phantoms for femoral and carotid artery waveforms are shown in Section IV and demonstrates the possibilities with the approach.

\section{VECTOR VELOCITY ACQUISITION AND ESTIMATION}

The blood velocity vector can be estimated along a measurement line using the TO method described in [6], [8], [11].

In TO vector flow imaging (VFI) a lateral oscillation with a wavelength of $\lambda_{x}$ is formed by using a receive apodization with two peaks. The wavelength depends on the emit focus, receive apodization function, and the interrogation depth. The TO wavelength is determined from [6], [12]:

$$
\lambda_{x}=\frac{2 \lambda D}{P_{d}}=\frac{2 \lambda D}{N_{d} P_{i}}=2 \lambda F_{\# T O},
$$

where $\lambda$ is the normal axial wavelength, $D$ is the depth, and $P_{d}$ is the distance between the two peaks in the apodization function. The transducer pitch is $P_{i}$ and the number of elements between the peaks is $N_{d}$. The TO F-number is denoted $F_{\# T O}=$ $\frac{D}{P_{d}}$. In DTO a received beam orthogonal to the ultrasound propagation direction is formed for the velocity estimation 
[10]. The beam is Hilbert transformed in the spatial direction and the velocity estimated from the complex signal in both the axial and lateral directions [8], [10]. The velocity angle and magnitude can then be determined for each time point and along the depth direction. In DTO the lateral wavelength can be estimated essentially making it self calibrating, and it also increases the accuracy of the estimates.

The method suggested here continuously emits ultrasound pulses in one direction, and the velocity as a function of time is continuously estimated. A number of advantages compared to spectral estimation can be gained from this as described below.

\section{A. Expanded velocity range and accuracy}

In a conventional ultrasound system using the autocorrelation approach [13] the maximum detectable velocity is [2]:

$$
v_{\max }=\frac{\lambda f_{p r f}}{4},
$$

where $f_{p r f}$ is the pulse repetition frequency. For the TO approach the maximum velocity possible to estimate is determined by the lateral wavelength and the pulse repetition frequency as [8]:

$$
v_{\text {max }}=\frac{\lambda_{x} f_{\text {prf }}}{4}=\frac{\lambda f_{\text {prf }}}{2} \frac{D}{P_{d}}=\frac{\lambda f_{\text {prf }}}{2} F_{\# T O} .
$$

$\lambda_{x}$ can be varied by changing $P_{d}$ in (1) or $F_{\# T O}$. Usually a value of $F_{\# T O}=2$ can be used, but higher values can also be selected for a penalty in resolution. For large velocities a small value of $P_{d}$ can be selected to yield a $\lambda_{x}$ four to eight times $\lambda$, thus, giving a maximum velocity 4 to 8 times larger than in a conventional system. The velocity can also be estimated with a low bias as described in [10] when a sufficient amount of data is available. For low velocities $P_{d}$ can be increased to make estimation better, as the lateral oscillation period is adapted to low velocity estimation by having a low $\lambda_{x}$. The continuous data also makes it possible to average over longer times compared to normal VFI to yield a low variance, as this is proportional to $1 / N$, where $N$ is the number of emissions.

The variation in $P_{d}$ is made during receive beamforming, and it is possible to have several different receive apodization functions to estimate low and high velocities simultaneously from the same data. It is also possible to adapt the apodization over time to yield both high systolic velocities as well as low diastolic velocities for the same data. Acquiring the full RF data before beamforming also makes it possible to retrospectively change the velocity range automatically by first estimating with a high maximum velocity range and then subsequently modify the apodization to reduce the maximum velocity.

The approach avoids the spectral broadening in the usual spectral estimates from the windowing [2], [5], and the methods can, thus, yield truly quantitative data with automatic angle estimation, as both the axial and transverse velocities are estimated.
It is also possible to display both the TO velocity estimates, the normal spectrogram, and the transverse spectrogram as described in [14] from the same data simultaneously.

\section{B. Displaying velocity profiles and measurements}

The velocities can be estimated as function of depth and time for the continuous data. The velocity profile can be shown as either arrows superimposed on the B-mode image or in other time-depth-velocity displays [7]. It also gives the possibility of showing either velocity magnitude or angle as a function of time or depth.

The velocity profile data makes it possible to directly calculate the volume flow for the vessel. The boundaries of the vessel is marked or estimated and the center position of the vessel is determined. Assuming a circular vessel, the volume flow $Q$ is found by integrating the velocities weighted by the area at the given depth as [15]:

$$
Q=\pi \Delta r \sum_{n=-N / 2}^{N / 2}|n| \vec{v}(n) \cdot \vec{e},
$$

where $\Delta r$ is the radial sampling interval, $N$ is the number of intervals within the vessel, $\vec{v}(n)$ is the velocity at sample $n$, and $\vec{e}$ is the unit vector for the plane to find the volume flow through. Elliptical vessel dimensions can be determined from the B-mode image perpendicular to the velocity view to find the major $d_{2}$ and minor axis $d_{1}$. For elliptical vessels the volume flow is then multiplied by $d_{2} / d_{1}$.

The estimation of velocity angle as a function of time can also reveal whether the flow is laminar or not. A stable angle over time shows that laminar flow is found. Deviations from the mean angle is an indication of disturbed or turbulent flow. The angle for a fully turbulent flow will randomly fluctuate between $-\pi$ to $+\pi$ with a rectangular probability density, which has a variance of $\sigma_{\Theta}^{2}=(2 \pi)^{2} / 12=\pi^{2} / 3$. An index $T_{i}$ for indicating whether the flow is turbulent could be:

$$
T_{i}=\sqrt{\frac{\hat{\sigma}_{\Theta}^{2}}{\pi^{2} / 3}}=\sqrt{\frac{\frac{1}{N_{\Theta}} \sum_{n=1}^{N_{\Theta}}(\hat{\Theta}(n)-E\{\hat{\Theta}(n)\})^{2}}{\pi^{2} / 3}}
$$

where $\Theta$ is the beam to flow angle, $\hat{\Theta}(n)$ is the velocity angle estimates, $\hat{\sigma}_{\Theta}^{2}$ is the estimated angle variance, and $E\{\}$ denotes mean value. $N_{\Theta}$ is the number of estimates averaged over in either time or space or both. A value of zero indicates a fully laminar flow with no angle variation, whereas a value approaching one is for fully turbulent flow. The index can be found over time and averaged, and it also can be averaged over space. It can further be used for displaying a turbulence map.

\section{Estimation of stenoses degree}

The degree of stenosis in a vessel can be found from the ratio between velocities measured before and at the stenosis. The DTO method has a higher maximum detectable velocity range than for spectral estimation, and it is therefore possible to measure at two different places intermixed using two focused emissions, and still have a sufficient velocity range 
for making a reliable index. The beams are then placed at the stenosis and before it, and the velocity ratio is then determined. Assuming a parabolic velocity profile, the stenosis degree calculated as the reduction in vessel diameter can be determined as:

$$
s_{d}=1-\sqrt{\frac{v_{2}}{v_{1}}},
$$

where $v_{1}$ is the velocity measured at the stenosis and $v_{2}$ is the velocity measured at the non-stenosed part of the vessel. The velocities can be either the mean, peak, or instantaneous velocity, or averaged values of these over time and/or space.

\section{MEASUREMENT SETUP}

A BK Medical 8820e convex array transducer was employed and VFI in a single direction was interleaved with a B-mode image implemented on the SARUS experimental scanner [16]. An active aperture of 64 elements was used during transmit for both sequences. The focal point was at $42 \mathrm{~mm}(F \#=2)$ for the B-mode and $105.6 \mathrm{~mm}(F \#=5)$ for VFI. A single cycle excitation was used for B-mode imaging and four cycles for flow imaging with a center frequency of $3 \mathrm{MHz}$. The transducer has 192 elements with $\lambda$ pitch and the B-mode image consisted of 129 lines. Pulsating flow was investigated using a CompuFlow 1000 pump (Shelley Medical Imaging Technologies, Toronto, Canada) capable of generating timevarying waveforms. It was connected to a wall-less, straight tube phantom with an internal diameter of $8 \mathrm{~mm}$ placed 38.2 $\mathrm{mm}$ from the probe at a beam-to-flow angle of $90^{\circ}$. The pump was set to produce waveforms mimicking that of a femoral and a carotid artery. The duration of one pump cycle was 840 $\mathrm{ms}$ and data for 10 cycles were acquired for 250 frames $(9.2$ seconds of data).

\section{RESULTS}

The velocity at the center of the vessel was estimated for all the cardiac cycles and the pulse period was automatically found from the autocorrelation of the velocity waveform. The different cycles were then aligned and the mean value and the standard deviation across cycles were estimated. They are shown for the femoral artery waveform in Fig. 1. From these estimates the mean angle was found to be $90.9^{\circ} \pm 8.20^{\circ}$ indicating a laminar flow as the turbulence index is $0.10 \pm 0.12$. The volume flow was found to be $1.29 \pm 0.26 \mathrm{~mL} /$ stroke. The pump was set to $1.15 \mathrm{~mL} /$ stroke and has a $3 \%$ accuracy $( \pm 0.035 \mathrm{~mL} / \mathrm{stroke})$. The peak velocity was found for all 9 full cycles and averaged to give a mean peak value of 0.24 $\mathrm{m} / \mathrm{s}$ with a range of 0.26 to $0.23 \mathrm{~m} / \mathrm{s}$ with an SD of $0.01 \mathrm{~m} / \mathrm{s}$. The velocity precision was also found to be $6.0 \%$ by averaging the variance of all 9 cycles.

Similar data were found for the carotid artery experiment, where the angle was $90.45^{\circ} \pm 3.10^{\circ}$, and the rest of the measures had similar values and precisions as for the femoral artery waveform.

The acquired data can also be used for visualizing the flow profile across the vessel as shown in Fig. 2 for the femoral

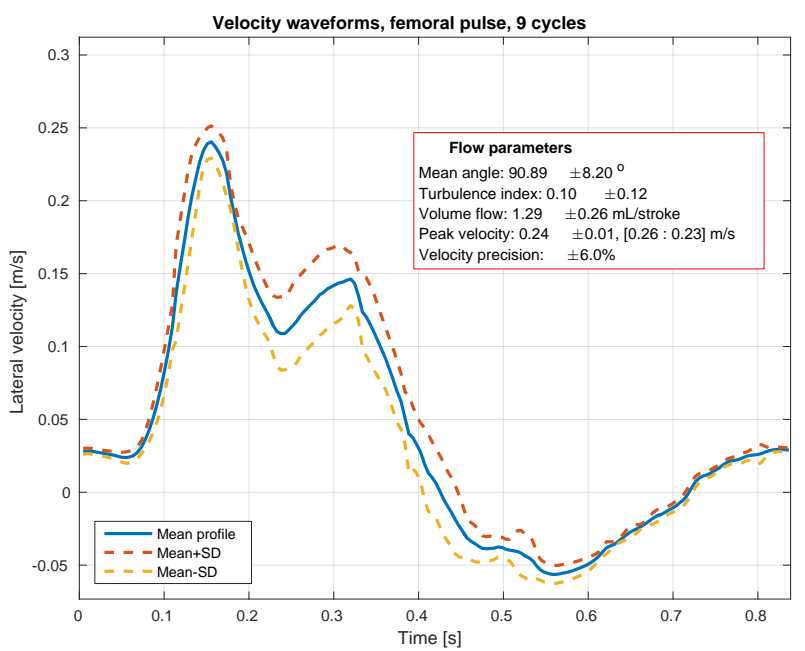

Fig. 1. Mean of velocity profiles over the cardiac cycle for the femoral artery.

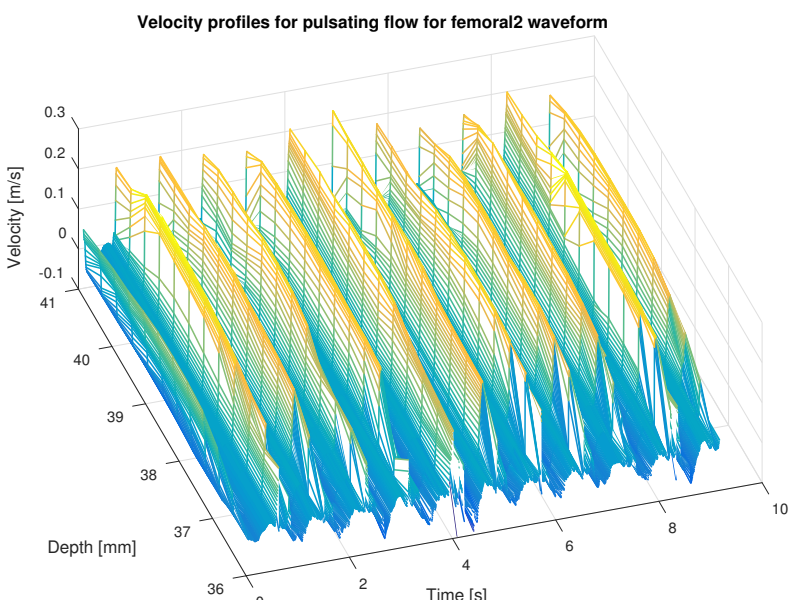

Fig. 2. Velocity profiles as a function of time and depth for the femoral artery data using 32 emissions.

data. Here the lateral velocity as a function of depth and time is visualized.

The TO method has for linear array probes been limited to interrogation depth of 5 to $6 \mathrm{~cm}$ due to the size of the active aperture, which is restricted when a vector flow image has to be made. This restricts the method to shallow vessels for linear probes. In the duplex mode the full aperture can be used and a lower F-number can be maintained over a longer distance. This is illustrated in Fig. 3 for a $12 \mathrm{~mm}$ vessel placed $156 \mathrm{~mm}$ from the probe with constant flow. The estimates were found using 32 emissions, and the beam-to-flow angle was $90^{\circ}$. For this situation a relatively low SD of $8.31 \%$ can be maintained along with a modest bias. The relative SD for the lateral velocity component for a number of deeper lying vessels are shown in Fig. 4 for beam-to-flow angles of $90^{\circ}$, $75^{\circ}$, and $60^{\circ}$.

\section{DISCUSSION}

Duplex VFI can be used for interrogating vessels down to a depth of at least $160 \mathrm{~mm}$ with a SD and bias of less than 

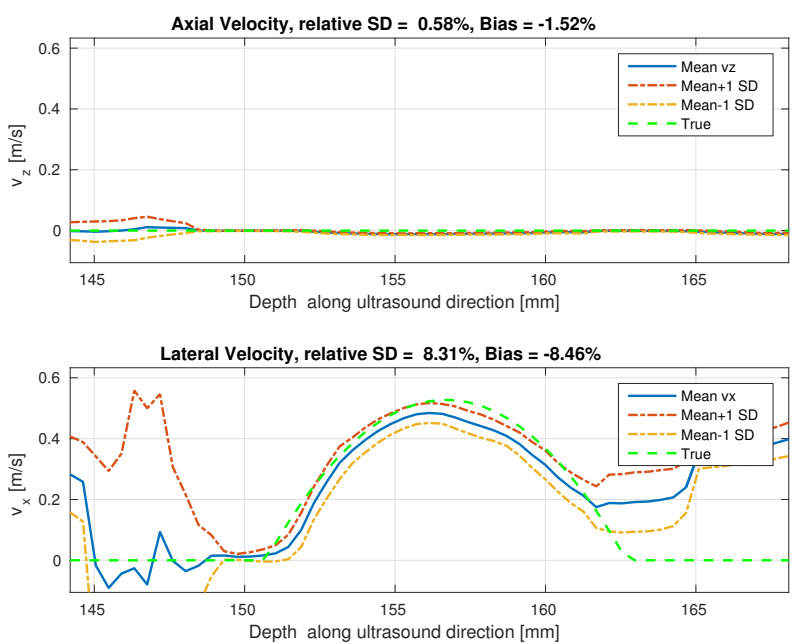

Fig. 3. Mean velocity profiles as a function of depth for stationary, parabolic flow in a vessel $156 \mathrm{~mm}$ from the probe surface. Thirty-two emissions were used for the estimation.
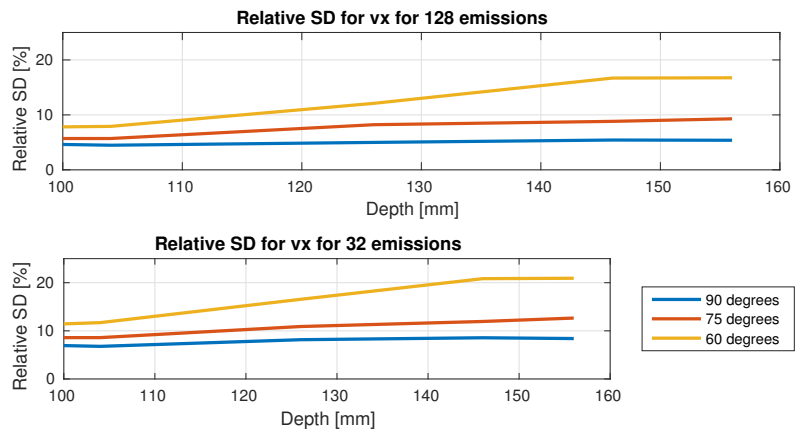

Fig. 4. Relative SD for the lateral velocity component for different vessel depths and beam to flow angles.

$10 \%$. At this depth it can be very difficult to angulate the ultrasound beam, thus, precluding the use of spectral velocity systems for beam-to-flow angles larger than $70^{\circ}$. Duplex VFI therefore gives new possibilities for investigating the vascular system. These new possibility comes from the employment of the full aperture of the 192 element convex probe and the continuous data available. Averaging over 32 to 128 emissions lowers the SD and using the DTO method lowers the bias to less than $10 \%$. For the pulsating examples shown for a more shallow vessel, the precision was around $6 \%$ for the complex pulsating flow including the inaccuracy of the pump generating the flow. The angle could also be determined within $\pm 8.2^{\circ}$ for the femoral artery and $\pm 3.1^{\circ}$ for the carotid artery waveform. Automatically aligning the estimates from 9 pulse beats gave the possibility of estimating mean angle, peak velocity, and volume flow along with the precisions of the measure. A turbulence index could also be found from the angle estimate and gives an indication whether the flow is laminar in one direction or disturbed over the cardiac cycle. All measures and indices are automatically obtained without user intervention and, thus, gives a system which is easier to use for inexperienced users and give more consistent results with an estimate of their precisions.

\section{CONCLUSION}

Making an imaging mode with continuous emission and DTO vector velocity estimation can give major benefits to measurements of flow quantities in ultrasound. It can replace the spectral display with a higher accuracy and avoids errors from both velocity angle deviations and spectral broadening. This give rise to a more easy, quantitative, and accurate measures of e.g. stenosis degree, volume flow, and turbulence index. The method can also during receive beamforming be adapted to the velocity range needed for both high and low velocities.

\section{ACKNOWLEDGEMENT}

This work was supported by grant 82-2012-4 from the Danish Advanced Technology Foundation and by B-K Ultrasound Aps, Herlev, Denmark.

\section{REFERENCES}

[1] D. H. Evans and W. N. McDicken, Doppler Ultrasound, Physics, Instrumentation, and Signal Processing. New York: John Wiley \& Sons, 2000.

[2] J. A. Jensen, Estimation of Blood Velocities Using Ultrasound: A Signal Processing Approach. New York: Cambridge University Press, 1996.

[3] J. Udesen, M. B. Nielsen, K. R. Nielsen, and J. A. Jensen, "Examples of in-vivo blood vector velocity estimation," Ultrasound Med. Biol., vol. 33, pp. 541-548, 2007.

[4] A. H. Brandt, K. L. Hansen, M. B. Nielsen, and J. A. Jensen, "Velocity estimation of the main portal vein with transverse oscillation." in Proc. IEEE Ultrason. Symp. IEEE, 2015, pp. 1-4.

[5] V. L. Newhouse, E. S. Furgason, G. F. Johnson, and D. A. Wolf, "The dependence of ultrasound Doppler bandwidth on beam geometry," IEEE Trans. Son. Ultrason., vol. SU-27, pp. 50-59, 1980.

[6] J. A. Jensen and P. Munk, "A new method for estimation of velocity vectors," IEEE Trans. Ultrason., Ferroelec., Freq. Contr., vol. 45, pp. 837-851, 1998.

[7] S. Ricci, L. Bassi, and P. Tortoli, "Real-time vector velocity assessment through multigate Doppler and plane waves," IEEE Trans. Ultrason., Ferroelec., Freq. Contr., vol. 61, no. 2, pp. 314-324, 2014.

[8] J. A. Jensen, "A new estimator for vector velocity estimation," IEEE Trans. Ultrason., Ferroelec., Freq. Contr., vol. 48, no. 4, pp. 886-894, 2001.

[9] J. Udesen and J. A. Jensen, "Investigation of Transverse Oscillation Method," IEEE Trans. Ultrason., Ferroelec., Freq. Contr., vol. 53, pp. 959-971, 2006.

[10] J. A. Jensen, "Improved vector velocity estimation using directional transverse oscillation," in Proc. IEEE Ultrason. Symp. IEEE, 2015, pp. $1-4$.

[11] M. E. Anderson, "Multi-dimensional velocity estimation with ultrasound using spatial quadrature," IEEE Trans. Ultrason., Ferroelec., Freq. Contr., vol. 45, pp. 852-861, 1998.

[12] J. W. Goodman, Introduction to Fourier optics, 2nd ed. New York: McGraw Hill Inc., 1996.

[13] C. Kasai, K. Namekawa, A. Koyano, and R. Omoto, "Real-Time TwoDimensional Blood Flow Imaging using an Autocorrelation Technique," IEEE Trans. Son. Ultrason., vol. 32, pp. 458-463, 1985.

[14] J. A. Jensen, "Transverse spectral velocity estimation," IEEE Trans Ultrason., Ferroelec., Freq. Contr., vol. 61, no. 11, pp. 1815-1823, november 2014.

[15] J. Jensen, J. B. Olesen, M. B. Stuart, P. M. Hansen, M. B. Nielsen, and J. A. Jensen, "Vector velocity volume flow estimation: Sources of error and corrections applied for arteriovenous fistulas," Ultrasonics, vol. 70, pp. 136-146, 2016.

[16] J. A. Jensen, H. Holten-Lund, R. T. Nilsson, M. Hansen, U. D. Larsen, R. P. Domsten, B. G. Tomov, M. B. Stuart, S. I. Nikolov, M. J. Pihl, Y. Du, J. H. Rasmussen, and M. F. Rasmussen, "SARUS: A synthetic aperture real-time ultrasound system," IEEE Trans. Ultrason., Ferroelec., Freq. Contr., vol. 60, no. 9, pp. 1838-1852, 2013. 